\title{
Hippocampus Dysfunction May Explain Symptoms of Fibromyalgia Syndrome
}

\author{
Yasser Emad ${ }^{1,2}$, Yasser Ragab ${ }^{3,4}$, Alaaabou-Zeid ${ }^{5}$, Johannes J. Rasker ${ }^{6}$ \\ Departments of ${ }^{1}$ Rheumatology, ${ }^{3}$ Radiology and ${ }^{5}$ Public Health, Cairo University, Cairo, Egypt, Departments of ${ }^{2}$ Rheumatology and ${ }^{4}$ Radiology, \\ Dr. Erfan and Bagedo General Hospital, Jeddah, Saudi Arabia, ${ }^{6}$ Department of Psychology, Health and Technology, Faculty of Behavioural, \\ Management and Social Sciences, University of Twente, Enschede, The Netherlands
}

\section{Abstract}

In fibromyalgia (FM) a combination of symptoms frequently exists, including impaired cognition, memory changes, widespread pain, and disrupted sleep rhythm, with no sufficient explanations for these bizarre symptoms. Many theories have been developed and it is still not clear whether FM is a chronic stress syndrome caused by many factors, including society or just a part of the spectrum of chronic pain. FM is the second most common rheumatic disorder behind osteoarthritis and, though still widely a puzzling syndrome, is now considered to be a central nervous system disorder, which is responsible for amplified and intensive pain. Over the years, three main ideas of hippocampal function have dominated the literature: Response inhibition, episodic memory, and spatial cognition. Given that the hippocampus plays integral roles in memory, cognition, and sleep regulations the later functions may be influenced by prolonged stress know to exist in FM syndrome. Moreover the hippocampus is an integral component of the limbic system, and as such may contribute to the negative affect and avoidance motivation experienced during pain experience and chronic stress. The aim of the current review is to focus on the physiological roles of the hippocampus and its relation to symptoms production in FM syndrome.

Keywords: Functional magnetic resonance imaging, fibromyalgia, nervous system

Fibromyalgia syndrome (FMS) is a chronic pain syndrome, characterised by widespread musculoskeletal pain with diffuse tenderness at multiple tender points. Despite intense investigations, the pathophysiology of fibromyalgia (FM) remains elusive. Evidence shows that it could be due to the changes in either the peripheral or central nervous system (CNS). For the CNS changes, alterations in the high brain area of FM patients have been investigated, but the definite mechanisms are still unclear. Magnetic resonance imaging (MRI) and functional MRI have been used to gather evidence regarding the changes of brain morphologies and activities in FM patients. Nevertheless, due to few studies, limited knowledge for alterations in brain activities in FM is currently available. ${ }^{[1]}$

A leading paradigm regarding the pathogenesis of FMS (and similar functional disorders) focuses on the concept of central sensitisation. This concept describes a situation in which there is an increased sensitivity of the CNS to the processing and transmission of pain, leading to the development of clinical phenomena such as allodynia and hyperalgesia. ${ }^{[2]}$

\begin{tabular}{|l|l|}
\hline \multicolumn{2}{|c|}{ Access this article online } \\
\hline Quick Response Code: & \multicolumn{1}{|c|}{ Website: } \\
& www.hamdanjournal.org \\
& \\
&
\end{tabular}

The hippocampus plays a role in memory and cognitive $\mathrm{e}^{[3]}$ functions that may be influenced by prolonged stress. The hippocampus also inhibits brain centres associated with the stress response, i.e., the hypothalamic paraventricular nucleus, central amygdala and locus coeruleus. ${ }^{[4]}$ The hippocampus is an integral component of the limbic system, and as such may contribute to the negative affect and avoidance motivation experienced during pain. A substantial body of evidence indicates that the hippocampus processes pain-related information, and some hippocampal neurons respond exclusively following noxious stimulation. ${ }^{[5]}$ Moreover, the hippocampus is vulnerable to damage and is particularly sensitive to the effects of adrenal glucocorticoids secreted during chronic stress. The damaging actions of glucocorticoids

Address for correspondence: Prof. Yasser Emad, Dr. Erfan and Bagedo General Hospital King, Fahd Street, P. 0. Box 6519 Jeddah 21452, Saudi Arabia. E-mail: yasseremad68@gmail.com

This is an open access journal, and articles are distributed under the terms of the Creative Commons Attribution-NonCommercial-ShareAlike 4.0 License, which allows others to remix, tweak, and build upon the work non-commercially, as long as appropriate credit is given and the new creations are licensed under the identical terms.

For reprints contact: reprints@medknow.com

How to cite this article: Emad Y, Ragab Y, Alaaabou-Zeid, Rasker JJ. Hippocampus dysfunction may explain symptoms of fibromyalgia syndrome. Hamdan Med J 2018;11:166-8. 
Emad, et al.: Hippocampus dysfunction and fibromyalgia symptoms

under such conditions have been termed allostatic load. ${ }^{[6]}$ Thus, understanding the status of the hippocampus in patients with FM might prove valuable in guiding future investigations, leading to effective therapy. ${ }^{[7]}$

The hippocampus participates in nociception, a function positively correlated with the activity of hippocampal $N$-methyl-D-aspartate (NMDA). Several stress-related hormones are known to enhance the activity of hippocampal NMDA receptors, increasing excitatory neurotransmission within the hippocampus. ${ }^{[7]}$ Blocking NMDA receptors in the hippocampal formation reduces nociceptive behaviours; this in turn supports the hypothesis that the hippocampal formation is involved in pain-related neural processing and expression of pain-related behaviours. ${ }^{[5]}$

Regional brain metabolism can be monitored by specific metabolic markers using proton magnetic resonance spectroscopy (1H-MRS). MRS has been used for the study of many physiological and pathological processes in the brain and elsewhere. With 1H-MRS, one can detect cerebral metabolites in vivo, most commonly $N$-acetyl aspartate (NAA) and choline (Cho) and creatine $(\mathrm{Cr})$-containing compounds. NAA is described as a neuron marker, because it is found at high concentrations almost exclusively in neurons, but is virtually undetectable in various other cell types, including glial cells. ${ }^{[7]}$

In a case-controlled study, we investigated Hippocampus by using $1 \mathrm{H}-\mathrm{MRS}$; our hypothesis was that hippocampal dysfunction may be responsible, at least in part, for symptoms seen in patients with FMS. We measured different metabolites NAA and Cho and Cr within the hippocampus in patients with FM and compared the findings with those in healthy controls. In addition we evaluated cognitive function by mini-mental state examination (MMSE) to assess aspects of cognitive functions among patients and controls. Depression assessment was carried out by using the Hamilton depression, while sleep assessment was assessed by structured sleep interview Diagnostic and Statistical Manual of Mental Disorders-IV11, using a sleep diary for 2 weeks.

In our study, we observed that, NAA levels of the right and left hippocampi were lower in the patients compared to controls ( $P=0.05$ and $P<0.003$, respectively), and therefore were statistically significant. Another statistically significant difference was observed in Cho levels in the right hippocampus, which were higher in the patient group; no difference between patient and control groups was found regarding other measured metabolites in hippocampi on both sides. ${ }^{[8]}$

Furthermore, significantly lower NAA/Cho ratios were observed in the right and left hippocampi in the patients compared to controls ( $P=0.001$ and $P=0.002$, respectively). Another significant difference was found between both groups regarding right and left $\mathrm{NAA} / \mathrm{Cr}$ ratios (right $\mathrm{NAA} / \mathrm{Cr}$ patients mean + standard deviation $(\mathrm{SD})=1.29 \mathrm{a} 0.53$, control $=2.321 .1$; $P=0.002$; left NAA/Cr patients mean $+\mathrm{SD}=1.64+0.69$, control $=2.611 .6 ; P=0.03)$, while $\mathrm{Ch} / \mathrm{Cr}$ ratios were not different on both sides. Moreover, significant correlations were found between language scores and right Cho and right Cr levels ( $P=0.041$ and $P=0.006$, respectively), while no other significant correlations were found between different aspects of cognitive functions as assessed by MMSE and other measured metabolites on both sides. ${ }^{[8]}$

Nevertheless, in similar work, Wood et al. ${ }^{[9]}$ investigated bilateral hippocampus of 16 female FM patients in comparison to 8 age- and gender-matched healthy controls using single-voxel 1H-MRS. Their results demonstrated a significant reduction in the ratio of $\mathrm{NAA} / \mathrm{Cr}$ in $\mathrm{FM}$ patients versus matched controls specifically in the right temporal lobe from a voxel centred on the right hippocampus (patient vs. control, mean \pm standard deviation: $1.20 \pm 0.13$ vs. $1.34 \pm 0.10$, $P=0.03$ ). Moreover, correlation analysis demonstrated a significant negative correlation between patient scores on the FM Impact Questionnaire and NAA/Cr ratio within the right hippocampus (Spearman's rank correlation, $r=-0.681$, $P=0.018$ ). Their findings indicate that FM is associated with brain metabolite abnormalities within the right hippocampus that correlate with FM symptoms.

To our knowledge, this is the first report that addresses significantly lower NAA, reduced NAA/Cr ratios, and higher Cho levels in the hippocampus as assessed by single-voxel $1 \mathrm{H}-\mathrm{MRS}$ among patients with FM, compared with controls. Lower hippocampal NAA levels suggest neuronal or axonal metabolic dysfunction, or some combination of these processes. Yet, neuronal loss within the hippocampus was not studied in our series, and this should be assessed in further studies, in order to elucidate whether the reduction in NAA level reflects actual neuronal loss due to atrophic changes within the hippocampus. We suggest that hippocampal dysfunction may be in part responsible for some of the phenomena associated with FM. Our observations are still preliminary, and further studies in larger numbers of patients are needed. Our findings may indicate ways to adoption of new therapeutic strategies for the treatment of patients with this puzzling syndrome.

\section{Financial support and sponsorship}

Nil.

\section{Conflicts of interest}

There are no conflicts of interest.

\section{REFERENCES}

1. Sawaddiruk P, Paiboonworachat S, Chattipakorn N, Chattipakorn SC. Alterations of brain activity in fibromyalgia patients. J Clin Neurosci 2017;38:13-22.

2. Ablin JN. Centralized pain and fibromyalgia: What do we mean when we say "it's all in your head"? Harefuah 2017;156:762-6.

3. Hazemeijer I, Rasker JJ. Fibromyalgia and the therapeutic domain. A philosophical study on the origins of fibromyalgia in a specific social setting. Rheumatology (Oxford) 2003;42:507-15.

4. Wood PB. Fibromyalgia syndrome: A central role for the hippocampus: A theoretical construct. J Musculoskelet Pain 2004;12:19-26. 
5. McKenna JE, Melzack R. Blocking NMDA receptors in the hippocampal dentate gyrus with AP5 produces analgesia in the formalin pain test. Exp Neurol 2001;172:92-9.

6. McEwen BS. Plasticity of the hippocampus: Adaptation to chronic stress and allostatic load. Ann N Y Acad Sci 2001;933:265-77.

7. Schuff N, Amend DL, Knowlton R, Norman D, Fein G, Weiner MW, et al. Age-related metabolite changes and volume loss in the hippocampus by magnetic resonance spectroscopy and imaging. Neurobiol Aging
1999;20:279-85.

8. Emad Y, Ragab Y, Zeinhom F, El-Khouly G, Abou-Zeid A, Rasker JJ, et al. Hippocampus dysfunction may explain symptoms of fibromyalgia syndrome. A study with single-voxel magnetic resonance spectroscopy. J Rheumatol 2008;35:1371-7.

9. Wood PB, Ledbetter CR, Glabus MF, Broadwell LK, Patterson JC $2^{\text {nd }}$. Hippocampal metabolite abnormalities in fibromyalgia: Correlation with clinical features. J Pain 2009;10:47-52. 